\title{
Dynamics of the low-lying pseudoscalar and vector mesons
}

\section{Terschlüsen*}

Department of Physics and Astronomy, Uppsala University, Sweden

E-mail: carla.terschluesen@physics.uu.se

\section{S. Leupold}

Department of Physics and Astronomy, Uppsala University, Sweden

\section{M.F.M. Lutz}

GSI, Darmstadt, Germany

The decays of low-lying vector mesons into a pseudoscalar meson and a dilepton and of the $\eta^{\prime}$ meson into an $\omega$-meson and a dielectron are calculated using a counting scheme which treats both the low-lying pseudoscalar mesons and the low-lying vector mesons on equal footing. All required parameters can be calculated from other decays and, hence, one has predictive power for the considered decay rates and form factors. Furthermore, the calculated partial decay widths are in good agreement with the available experimental data.

International Winter Meeting on Nuclear Physics,

21-25 January 2013

Bormio, Italy

\footnotetext{
* Speaker.
} 


\section{Introduction}

Describing strong interactions in hadronic reactions and decays is an open challenge in hadron physics. Due to the running coupling strength in QCD, perturbation theory cannot be used for lowenergy hadron physics. Instead, effective theories can be used which include hadrons instead of quarks and gluons as relevant degrees of freedom. For the energy regime of the pseudoscalar octet, pions, kaons and $\eta$-meson, the dynamics in the threshold region can be successfully described by chiral perturbation theory (ChPT) [1,2]. ChPT is not applicable to describe dynamics in the energy range of the hadronic resonances $\rho-, \omega-, K^{*}-, \phi$ - and $\eta^{\prime}$-meson since these particles are treated as heavy.

Electromagnetic reactions of hadrons can be described efficiently with dynamical vector meson degrees of freedom according to the vector-meson-dominance conjecture [3]. Many reactions are phenomenologically successfully described by the standard vector-meson-dominance model (VMD) but a consistent picture cannot be generated within this model. VMD fails, e.g., to describe the $\omega$ - $\pi^{0}$-transition form factor, i.e. the transition form factor for the decay of an $\omega$-meson into a neutral pion and a dilepton [4]. Such transition form factors will be studied in the following using an effective Lagrangian with explicit vector-meson fields [5].

In $[6,5]$, a counting scheme was proposed which treats both the low-lying pseudoscalar and vector mesons as soft, i.e. of the order of a typical momentum $Q$. Hence, the counting rules are given as

$$
m_{P}, m_{V} \sim Q, D_{\mu} \sim Q
$$

for masses of pseudoscalar and vector mesons, $m_{P}$ and $m_{V}$, respectively, and a covariant derivative $D_{\mu}$. According to the hadrongenesis conjecture all other genuine quark-antiquark states are much higher in mass as schematically displayed in Fig. 1. This mass gap quantified by $\Lambda_{\text {hard }}$ provides a dimensionless expansion parameter $Q / \Lambda_{\text {hard }}$. All other observed low-lying mesons are dynamically generated from interactions of low-lying pseudoscalar and vector mesons $[6,7,8,9,10,5]$ which justifies the restriction to pseudoscalar and vector mesons as the only relevant degrees of freedom.

\section{Leading-order Lagrangian}

Accoring to the counting scheme (1.1), the leading-order Lagrangian relevant for the decays $V \rightarrow P \gamma^{*}$ and $P \rightarrow V \gamma^{*}$ of a vector meson $V$ and a pseudoscalar meson $P$, respectively, is given by

$$
\begin{aligned}
\mathscr{L}= & -\frac{h_{A}}{16 f} \varepsilon^{\mu v \alpha \beta} \operatorname{tr}\left\{\left[\Phi_{\mu v}, \partial^{\tau} \Phi_{\tau \alpha}\right]_{+} \partial_{\beta} \Phi\right\}-\frac{b_{A}}{8 f} \varepsilon^{\mu v \alpha \beta} \operatorname{tr}\left\{\Phi_{\mu v}\left[\Phi, \chi_{0}\right]_{+} \Phi_{\alpha \beta}\right\} \\
& -\frac{m_{V}^{2} h_{H}}{4 f_{H}} \varepsilon^{\mu v \alpha \beta} \operatorname{tr}\left\{\Phi_{\mu v} \Phi_{\alpha \beta}\right\} \tilde{\eta}_{1}-e f_{V} \operatorname{tr}\left\{\Phi^{\mu v} Q\right\} \partial_{\mu} A_{v} \\
& +\frac{e f_{V} e_{H}}{f_{H}} \varepsilon^{\mu v \alpha \beta} \operatorname{tr}\left\{\Phi_{\mu v} Q\right\} \partial_{\alpha} A_{\beta} \tilde{\eta}_{1} .
\end{aligned}
$$




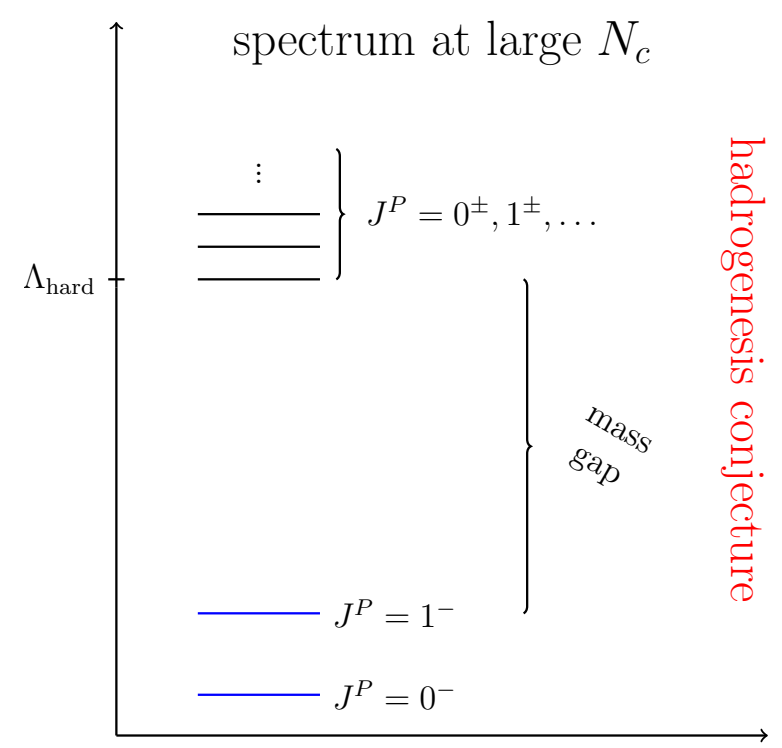

Figure 1: The meson spectrum of QCD in the limit of a large number of colors $N_{c}$ as conjectured in the hadrongenesis picture.

Hereby, the matrix $\Phi_{\mu v}$ describes the vector-meson nonet represented by antisymmetric tensor fields and $\Phi$ the pseudoscalar nonet,

$$
\begin{gathered}
\Phi_{\mu v}=\left(\begin{array}{ccc}
\rho_{\mu v}^{0}+\omega_{\mu v} & \sqrt{2} \rho_{\mu v}^{+} & \sqrt{2} K_{\mu \nu}^{+} \\
\sqrt{2} \rho_{\mu \nu}^{-} & -\rho_{\mu \nu}^{0}+\omega_{\mu v} & \sqrt{2} K_{\mu \nu}^{0} \\
\sqrt{2} K_{\mu \nu}^{-} & \sqrt{2} \bar{K}_{\mu \nu}^{0} & \sqrt{2} \phi_{\mu v}
\end{array}\right), \\
\Phi=\left(\begin{array}{ccc}
\pi^{0}+\frac{1}{\sqrt{3}} \eta_{8} & \sqrt{2} \pi^{+} & \sqrt{2} K^{+} \\
\sqrt{2} \pi^{-} & -\pi^{0}+\frac{1}{\sqrt{3}} \eta_{8} & \sqrt{2} K^{0} \\
\sqrt{2} K^{-} & \sqrt{2} \bar{K}^{0} & -\frac{2}{\sqrt{3}} \eta_{8}
\end{array}\right)+\tilde{\eta}_{1} \frac{f}{f_{H}} \sqrt{\frac{2}{3}} I_{3 \times 3} .
\end{gathered}
$$

Furthermore, $\chi_{0}=\operatorname{diag}\left(\mathrm{m}_{\pi}^{2}, \mathrm{~m}_{\pi}^{2}, 2 \mathrm{~m}_{\mathrm{K}}^{2}-\mathrm{m}_{\pi}^{2}\right)$ denotes the mass matrix, $Q=\operatorname{diag}(2 / 3,-1 / 3,-1 / 3)$ the quark-charge matrix and $A_{\mu}$ the photon field. The pion decay constant is given as $f=90 \mathrm{MeV}$, the vector-meson decay constant as $f_{V}=140 \mathrm{MeV}$, the generic vector-meson mass as $m_{V}=764$ $\mathrm{MeV}$ and the positron charge as $e=\alpha /(4 \pi)=0.303$.

The Lagrangian (2.1) allows only the $\tilde{\eta}_{1}$-state to couple directly to a vector meson and a photon, i.e. there exists only a $\tilde{\eta}_{1}-V-\gamma$ vertex described by the last term proportional to the parameter $e_{H}$ and no $P-V-\gamma$ vertex for any other pseudoscalar meson $P$. Therefore, only the decays $V \rightarrow \tilde{\eta}_{1} \gamma^{(*)}$ (see left-hand side in Fig. 2) and $\tilde{\eta}_{1} \rightarrow V \gamma^{(*)}$ can happen directly. Decays with an arbitrary pseudoscalar (including the $\tilde{\eta}_{1}$-state) as, e.g., $V \rightarrow P \gamma^{(*)}$ (see right-hand side in Fig. 2) can happen via a virtual vector meson, i.e. such decays consist of a $P-V-V^{*}$ vertex described by the first three terms in (2.1) proportional to $h_{A}, b_{A}$ and $h_{H}$ and a $V^{*}-\gamma^{(*)}$ vertex described by the term proportional to $e f_{V}$. The decay of a virtual photon into a dilepton is described by usual QED.

The pseudoscalar nonet $\Phi$ in (2.1) includes the non-physical singlet state $\tilde{\eta}_{1}$ and octet state $\eta_{8}$. Therewith, the physical fields $\eta$ - and $\eta^{\prime}$-meson are defined as linear combinations of the $\tilde{\eta}_{1}$ - and 

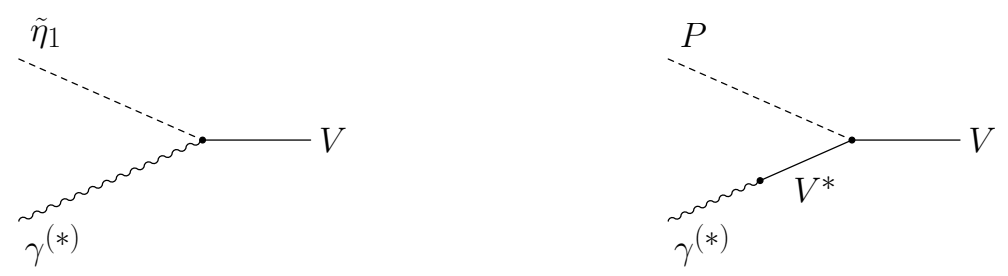

Figure 2: Feynman diagrams for the direct decay $V \rightarrow \tilde{\eta}_{1} \gamma^{(*)}$ (left) and the indirect decay $V \rightarrow P \gamma^{(*)}$ via a virtual vector meson $V^{*}$ for an arbitrary pseudoscalar meson $P$ (right).

$\eta_{8}$-state with a to be determined mixing angle $\theta$,

$$
\begin{aligned}
\eta & =-\tilde{\eta}_{1} \sin \theta+\eta_{8} \cos \theta, \\
\eta^{\prime} & =\tilde{\eta}_{1} \cos \theta+\eta_{8} \sin \theta
\end{aligned}
$$

Since the physical fields are defined as non-mixing, the leading-order mass and kinetic terms for pseudoscalar mesons [5] can be used to determine the mixing angle,

$$
\cos (2 \theta)=\frac{m_{\eta^{\prime}}^{2}+m_{\eta}^{2}-\frac{2}{3}\left(4 m_{K}^{2}-m_{\pi}^{2}\right)}{m_{\eta^{\prime}}^{2}-m_{\eta}^{2}},
$$

yielding $\theta=-10.7^{\circ}$. Alternatively, one can use this to calculate the $\eta^{\prime}$ - and $\eta$-meson mass as functions of the mixing angle, the experimental pion and kaon masses and the combination $m_{\eta^{\prime}}^{2}+$ $m_{\eta}^{2}$ of the experimental $\eta$ - and $\eta^{\prime}$-masses. For $|\theta| \leq 15^{\circ}$, the discrepancy between theoretical and experimental masses are less than $5 \%$ (see Fig. 3). Therefore, the mixing angle $\theta$ is used as an additional open parameter.

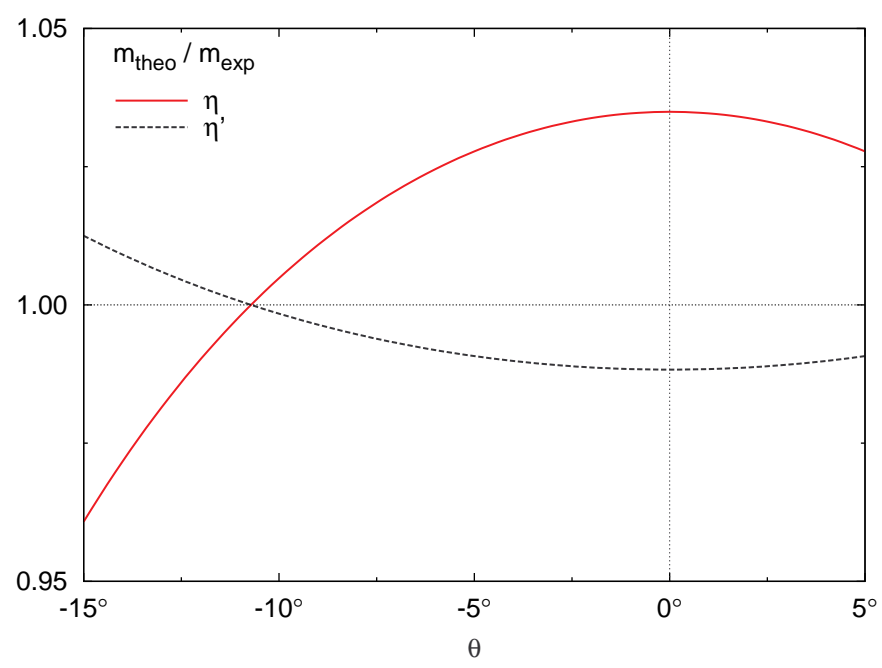

Figure 3: Ratio $m_{\text {theo }} / m_{\text {exp }}$ calculated with the relation (2.5) and the experimental values for the pion mass, the kaon mass and the mass combination $m_{\eta^{\prime}}^{2}+m_{\eta}^{2}$. 


\section{Parameter determination}

The Lagrangian (2.1) has six open parameters, $h_{A}, b_{A}, h_{H}, e_{H}, f_{H}$ and (implicitly) the $\eta-\eta^{\prime}$ mixing angle $\theta$. All parameters except $f_{H}$ can be determined from the five two-body decays

$$
\omega \rightarrow \pi^{0} \gamma, \omega \rightarrow \eta \gamma, \phi \rightarrow \eta \gamma, \phi \rightarrow \eta^{\prime} \gamma, \eta^{\prime} \rightarrow \omega \gamma
$$

Since the decay width depends only on the squared matrix element, the experimental values can still be described by four different parameter sets after fixing the parameter $h_{A}$ to be positiv. From those parameter sets, two sets with "resonable" parameters compared to previous calculations without the $\eta^{\prime}$-meson $[6,11,12]$ are chosen,

$$
\begin{aligned}
\theta & = \pm 2.0^{\circ}, \\
h_{A} & =2.33, \\
b_{A} & =0.16, \\
h_{H} & =0.14 \mp 0.19 f_{H} / f, \\
e_{H} & =-0.20 \mp 0.70 f_{H} / f .
\end{aligned}
$$

Note that only the mixing angle $\theta$ and the parameters $h_{H}$ and $e_{H}$ corresponding to the terms with explicit $\tilde{\eta}_{1}$-fields in the Lagrangian depend on the parameter set. The still open parameter $f_{H}$ will be varied between the pion decay constant $f$ and $\sqrt{2} f \approx f_{V}$. In the following, the parameter sets will be identified via the sign of the mixing angle.

For decays into dileptons, no additional parameters are needed and, therefore, one has predictive power.

\section{Results}

\subsection{Decay $\omega \rightarrow \pi^{0} l^{+} l^{-}$}

Due to isospin conservation, the decay $\omega \rightarrow \pi^{0} l^{+} l^{-}$is only possible via a virtual $\rho^{0}$-meson. Therefore, the standard VMD form factor is given by

$$
F_{\omega \pi^{0}}^{\mathrm{VMD}}(q)=\frac{m_{\rho}^{2}}{m_{\rho}^{2}-q^{2}}
$$

with the invariant dilepton mass $|q|$. Our calculations using the Lagrangian (2.1) yield an additional constant term compared to VMD,

$$
F_{\omega \pi^{0}}(q) \sim h_{A}+\frac{-\left(m_{\omega}^{2}+m_{\rho}^{2}\right) h_{A}+8 b_{A} m_{\pi}^{2}}{m_{\rho}^{2}-q^{2}}
$$

whereby the proportionality factor is fixed according to the normalization condition $F_{\omega \pi^{0}}(0)=1$. On the left-hand side of Fig. 4, the $\omega-\pi^{0}$ transition form factor (4.2) is plotted in comparison to the VMD prediction and data taken by the NA60 collaboration [4]. As already mentioned in the introduction, the VMD prediction fails to describe the data. Our calculation describes the data very 
well and misses only the last three data points. This is supported by a smaller reduced $\chi^{2}$ for the single-differential decay width calculated with (4.2),

$$
\chi_{\text {ourtheo. }}^{2} / N=1.8, \chi_{\mathrm{VMD}}^{2} / N=4.8 .
$$
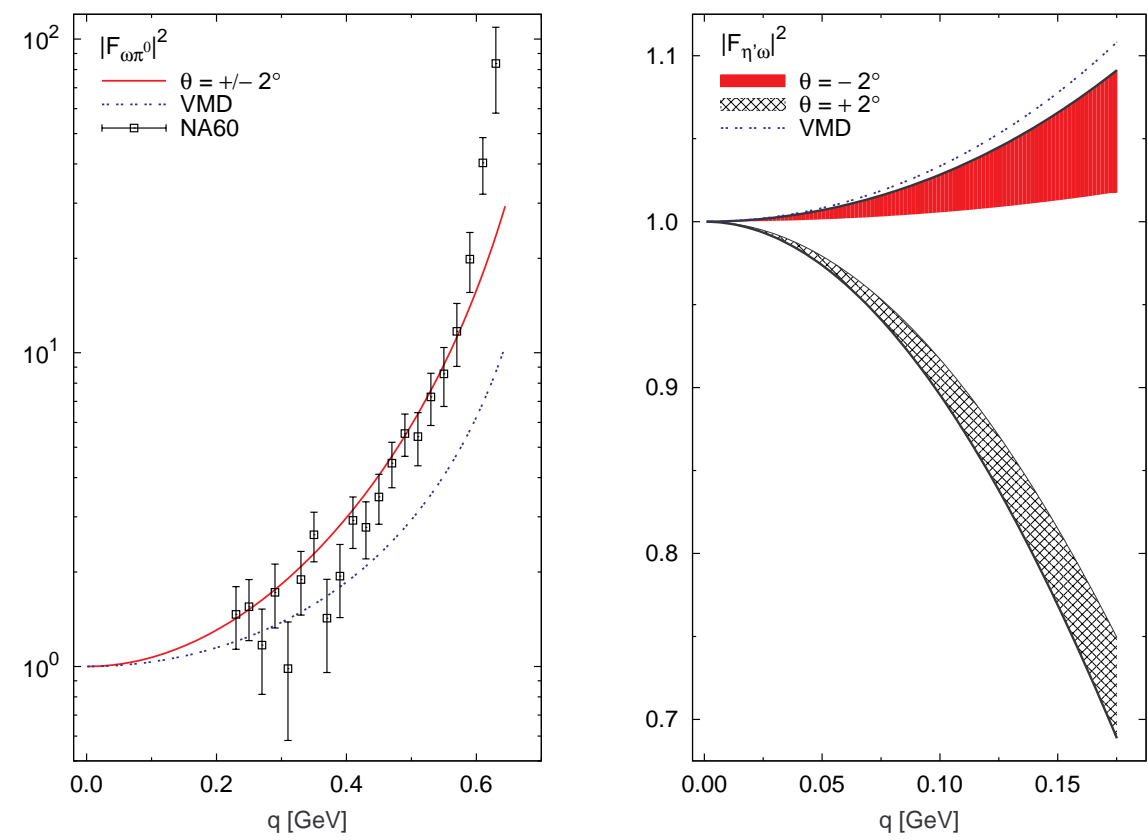

Figure 4: Left: Normalized form factor for the decay $\omega \rightarrow \pi^{0} l^{+} l^{-}$given by (4.2) (solid red line) in comparison to the VMD prediction (dashed blue line) and data taken by the NA60 collaboration for the decay into dimuons [4]. Right: Normalized form factor for the decay $\eta^{\prime} \rightarrow \omega e^{+} e^{-}$given by (4.5) with $f_{H} \in[f, \sqrt{2} f]$ (red / black for $\theta=-2^{\circ} /+2^{\circ}$ ) in comparison to the VMD prediction (dashed blue line).

In addition, the calculated decay widths for both the decay into a dimuon and into a dielectron agree very well with the experimental values,

$$
\begin{aligned}
\Gamma_{\omega \rightarrow \pi^{0} \mu^{+} \mu^{-}} & =(9.74 \pm 0.30) \cdot 10^{-7} \mathrm{GeV}, \\
\Gamma_{\omega \rightarrow \pi^{0} \mu^{+} \mu^{-}}^{\exp } & =(11.04 \pm 3.40) \cdot 10^{-7} \mathrm{GeV}, \\
\Gamma_{\omega \rightarrow \pi^{0} e^{+} e^{-}} & =(6.85 \pm 0.21) \cdot 10^{-6} \mathrm{GeV}, \\
\Gamma_{\omega \rightarrow \pi^{0} e^{+} e^{-}}^{\exp } & =(6.54 \pm 0.51) \cdot 10^{-6} \mathrm{GeV} .
\end{aligned}
$$

For an alternative calculation of the transition form factors $\omega / \phi \rightarrow \pi^{0} l^{+} l^{-}$see [13].

\subsection{Decay $\eta^{\prime} \rightarrow \omega e^{+} e^{-}$}

The decay $\eta^{\prime} \rightarrow \omega e^{+} e^{-}$can happen both directly $\left(e_{H}\right.$ term) or via a virtual $\omega$-meson. Additionally, one has to take $\eta-\eta^{\prime}$ mixing into account yielding the form factor

$$
F_{\omega \eta^{\prime}} \sim \hat{f}_{\eta_{8} \omega} \sin \theta+\frac{f}{f_{H}} \hat{f}_{\eta_{1} \omega} \cos \theta .
$$


The form factors for the formal decays of the octet and singlet states include again an additional constant term,

$$
\begin{aligned}
& \hat{f}_{\eta_{8} \omega}(q) \sim h_{A}+\frac{-2 m_{\omega}^{2} h_{A}+8 m_{\pi}^{2} b_{A}}{m_{\omega}^{2}-q^{2}}, \\
& \hat{f}_{\eta_{1} \omega}(q) \sim \sqrt{2}\left\{h_{A}-2 \sqrt{6} e_{H}+\frac{-2 m_{\omega}^{2} h_{A}+8 m_{\pi}^{2} b_{A}+4 \sqrt{6} m_{V}^{2} h_{H}}{m_{\omega}^{2}-q^{2}}\right\},
\end{aligned}
$$

compared to the VMD prediction,

$$
F_{\eta^{\prime} \omega}^{\mathrm{VMD}}(q)=\frac{m_{\omega}^{2}}{m_{\omega}^{2}-q^{2}} .
$$

The $\eta^{\prime}-\omega$ transition form factor is plotted on the right-hand side of Fig. 4 in comparison to the VMD prediction. Here, a clear deviation between the calculations with the two parameter sets for $\theta= \pm 2^{\circ}$ and an uncertainty caused by the range of $f_{H}$ can be observed. Nevertheless, for all calculations our results are (clearly) distinguishable from the VMD prediction.

Unfortunately, there is no experimental data for the decay $\eta^{\prime} \rightarrow \omega e^{+} e^{-}$available which could be used to determine the sign of the mixing angle and to restrict the range for $f_{H}$. We predict a branching ratio of

$$
\mathrm{BR}_{\eta^{\prime} \rightarrow \omega \mathrm{e}^{+} \mathrm{e}^{-}}=(1.69 \pm 0.56) \cdot 10^{-4}
$$

\section{Summary}

We applied a new counting scheme which treats both the low-lying pseudoscalar and vector mesons on the same footing to study the leading-order transition form factors of the transitions $\omega$ $\pi^{0}$ and $\eta^{\prime}-\omega$. The partial decays widths in leading order for the decay of an $\omega$-meson into a neutral pion and both a dimuon and a dielectron are in good agreement with the experimental data. The available experimental data for the $\omega$ - $\pi^{0}$-transition form factor is much better described with our approach than with standard VMD. Additionally, we predicted the leading-order branching ratio for the decay $\eta^{\prime} \rightarrow \omega e^{+} e^{-}$. Note that the decays presented here are only examples, the decays $\omega \rightarrow \eta l^{+} l^{-}, \phi \rightarrow \eta l^{+} l^{-}$and $\phi \rightarrow \eta^{\prime} e^{+} e^{-}$have been calculated in [5].

\section{References}

[1] J. Gasser and H. Leutwyler, Nucl. Phys. B250 (1985) 465.

[2] S. Scherer, Adv. Nucl. Phys. 27 (2003) 277.

[3] J.J. Sakurai, Currents and mesons (University of Chicago Press, Chicago, USA, 1969).

[4] R. Arnaldi et al., Phys. Lett. B277 (2009) 260.

[5] C. Terschlüsen, S. Leupold and M.F.M. Lutz, Eur. Phys. J. A48 (2012) 190.

[6] M.F.M. Lutz and S. Leupold, Nucl. Phys. A813 (2008) 96.

[7] M.F.M. Lutz and E. E. Kolomeitsev, Nucl. Phys. A730 (2004) 392. 
[8] M.F.M. Lutz and M. Soyeur, Nucl. Phys. A813 (2008) 14.

[9] I.V. Danilkin, L.I.R. Gil and M.F.M. Lutz, Phys. Lett. B703 (2011) 504.

[10] I.V. Danilkin, M.F.M. Lutz, S. Leupold, C. Terschlüsen, Eur. Phys. J. C73 (2013) 2358.

[11] S. Leupold and M.F.M. Lutz, Eur. Phys. A39 (2009) 205.

[12] C. Terschlüsen and S. Leupold, Phys. Lett. B691 (2010) 191.

[13] S.P. Schneider, B. Kubis and F. Niecknig, Phys. Rev. D86 (2012) 054013. 